\title{
In vivo study of biomechanical properties in psoriasis vulgaris: Effectiveness of sulfur spa therapy
}

\author{
Sergio Mazzulla ${ }^{1}$, Virgilia Nicoletta ${ }^{1}$, Ida Perrotta ${ }^{2}$, Stefania De Stefano ${ }^{3}$, Settimio Sesti ${ }^{1}$ \\ ${ }^{1}$ Department of Cellular Biology, University of Calabria, Rende, Italy \\ ${ }^{2}$ Department of Ecology, University of Calabria, Rende, Italy \\ ${ }^{3}$ Luigiane Thermae, Guardia Piemontese, Acquappesa, Italy \\ Email: mazzulla@unical.it
}

Received 1 January 2013; revised 2 February 2013; accepted 10 February 2013

\begin{abstract}
Psoriasis is a prolonged inflammation of the skin. The causes of psoriasis are still unclear. The treatment options depend on the severity of the disease and may include topical agents (such as topically-applied drugs, sulfur spa therapy, phototherapy) and systemic agents (orally or percutaneously administered). The aim of this study was to investigate the mechanical properties of the skin in psoriatic plaques before and after treatment with sulfur therapy (Thermae Luigiane, Guardia Piemontese-Acquappesa, Italy), in comparison with the skin of healthy subjects. The study has been performed on 20 psoriatic plaques (10 on upper arm, 10 on upper back) and 10 control subjects (healthy males aged $47 \pm 15$ ). The efficacy of sulfur therapy was observed through the evaluation of the biomechanical properties of the skin. Investigation was performed with a Skin meter, an instrument useful to determine the physiological parameters by the means of a suction method, a non-invasive in vivo suction. The apparatus was equipped with 2-mm measuring probe. Unlike the optical method, in our test the color and/or distribution of the skin microcirculation didn't interfere with the measurement. The evaluation of mechanical properties of the psoriatic plaques after treatment showed a significative recovery of the parameters analysed, with an increase of the elasticity parameters (Ur, Ua, Ua/Uf, Ur/Uf) and a decrease of the visco-elasticity module $(\mathrm{Uv} / \mathrm{Ue})$.
\end{abstract}

Keywords: Psoriasis; Sulfur Spa Therapy; Mechanichal Properties of Skin; Skin Meter

\section{INTRODUCTION}

Psoriasis is a chronic inflammatory and proliferative disorder of the skin clinically manifested as well-circumscribed, erythematous papules and plaques covered with silvery scales. The lesion location is generally on elbows, knees, scalp, lower back, face, palms, and feet soles [1]. The pathogenesis of this skin disease is still unclear. The treatment options depend on the severity of the disease and may consist in topical agents (drugs applied to the skin, phototherapy etc.) and/or systemic agents (orally or percutaneously administered). Although, specific systemic and environmental factors are known to influence the disease, its course is unpredictable and usually develops spontaneously with increase and exacerbations of lesions without discernible causes. Recent researches indicate that immune system dysfunction in the background of a genetic predisposition may be involved in the onset of the disease [2]. In a previous study we have shown the efficacy of a topic therapy with the use of an organic matrix, deriving from micro-flora, grown in shallow hyper thermal sulfurous water tubs of Guardia Piemontese-Acquappesa (CS) Italy. We actually reported on the attenuation of such psoriasis symptoms, as scales reduction of erythema and pruritus with decrease of lesions size and reddening intensity [3]. The organic matrix substances (carotenoids, retinoids and the unsaponificable fraction) present in micro-flora lipids naturally containing phytosterols and tocopherols, seem to possess not only antioxidant but also anti-inflammatory activities [4]. The organic matrix has been proven to exert protective effects on RBC membrane fluidity after the psoriatic skin lesion's treatment and also on the levels of lipid peroxidation [4,5]. Psoriasis is considered a life-long disease and no causal therapy for the disease is yet available, therefore the need for safe and efficacious long-term treatments is of major importance. Traditional systemic therapies for psoriasis include methotrexate [MTX], cyclosporin A, retinoids or PUVA therapy, which have a potential for long-term toxicity and may not always provide sufficient improvement of the disease $[6,7]$. Biological therapies (T-cell modulating agents (alefacept and efalizumab), the inhibitors of tumour necrosis factor-a (TNFa blockers, e.g. adalimumab, certolizumab, etanercept, golimumab, and infliximab), 
and the inhibitors of IL-12 and IL-23 (ustekinumab and briakinumab), have demonstrated efficacy in patients with psoriasis and are generally considered safe and well tolerated. However some rare but serious safety issues (i.e., demyelination, infection, tuberculosis, malignancy, lymphoma, cardiovascular outcomes, hepatitis, pregnancy, surgery, and vaccination) have been observed [8-10]. Taking into account the possibility that side effects might occur during long-term exposure to these drugs, the sulfur spa therapy combined with pharmacological agents may represent an useful alternative for the treatment of moderate psoriatic lesions. To achieve this purpose, we investigated the mechanical properties of the skin in psoriatic plaques of patients before and after treatment with sulfur spa therapy in comparison to the skin of healthy controls. Recently, a great deal of in vivo studies have been using a bioengineering approach to evaluate the mechanical properties of the epidermal tissue [11-13]. The instrument used herein for assessing dermal deformation is based on the measurements of skin parameter [13]. Progressive resistance to deformation, flexibility and elasticity must be adequately balanced to fulfill the ideal tensile properties of the skin. Furthermore, the parameters described are the functional expression of the molecular rearrangements of the skin components. The structural alteration of the skin can be ascribed to immune system dysfunction involving a complex interrelationship between hyperplastic epidermal keratinocytes and several immune cell types including $\mathrm{T}$ cells, neutrophils, dendritic cells and macrophages [14-18]. Therefore, the alteration in skin mechanics of the psoriatic plaques is thought to be due to increased skin thickness as a result of epidermal hyper proliferation and vasodilatation in the papillary dermis [13]. Nowadays biological treatments for psoriasis are gaining more and more popularity, and bath therapy with sulfur mineral water represents a safe and efficient support for integrated treatment to the conventional therapeutic modalities.

\section{MATERIAL AND METHODS}

\subsection{Subject}

The study has been performed on 10 normal weight male patients (age range $47 \pm 15$ ) with psoriasis plaques. A total of 20 psoriatic plaques was measured, of which 10 were localized on the extremities (arm) and 10 on the trunk (upper back). Ten comparable healthy subjects (males, age range $50 \pm 13$ ) as control group were also studied. Healthy subjects had no evident cutaneous damage. None of the subjects was alcohol or narcotics addict or had received any systemic therapy. Informed consensus for participation in the study was obtained from all the subjects.

\subsection{Sulfur Spa Therapy}

The water in use for treatment in psoriasis disease comes from two salt-bromine-iodine-sulphurous hyperthermal and thermal springs respectively at $43^{\circ} \mathrm{C}$ and $22^{\circ} \mathrm{C}$ from Luigiane Thermae of Guardia Piemontese (CS-Italy). Hyperthermal waters contain a very high rate of sulfur $(173 \mathrm{mg} / \mathrm{L})$. These waters are also used for baths, mud, inhalations and have resulted very effective in treating skin diseases, respiratory problems, orthopedic and arthrorheumatism. The therapeutic cycle of balneotherapy consists in one daily bath, with total immersion in individual bath tub, lasting 20 minutes at $35^{\circ} \mathrm{C}$, for 12 days and the assessment has been conducted at constant temperature and humidity $\left(25^{\circ} \mathrm{C}\right.$ and $40 \%$ relative humidity).

\subsection{Skin Meter Apparatus}

The elasticity, viscoelasticity, and distensibility of human skin is assessed with a "Skin meter", an instrument useful to determine the physiological parameters using the suction method. This measurement may be useful to evaluate the mechanical properties of psoriasis skin before and after bath therapy with sulfur mineral water. The stick of irrelevant mass is immersed in an electromagnetic field and causes a change of the reluctance of the circuitry, due to the linear displacement caused by the negative pressure applied on the skin. The stick, located in the probe circuitry, has been placed on the area of skin under examination. The probe of the electromechanical Skin-meter produces a continuous transduced signal that is accurately and easily evaluated by the instrument. This determination method, unlike the optical methods, is independent from color, skin microcirculation and skin irregularity. It allows us to appreciate directly the elastic deformation in the skin area analyzed and bounded (in a diameter of 2-mm) by the geometry of the probe (Figure 1).

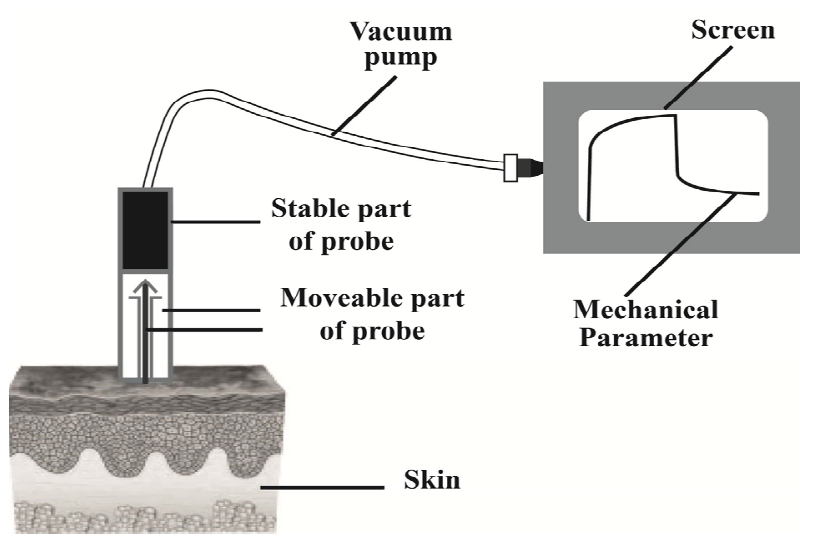

Figure 1. Representation of elastomer probe on human skin. 


\subsection{Skin Parameter}

The mechanical properties of the dermis were determined using a non-invasive, in vivo suction skin elasticity meter equipped with $2-\mathrm{mm}$ measuring probe. The time/strain mode was used with a $5 \mathrm{sec}$. application of a constant negative pressure of 400 mbar, followed by a 5 $\mathrm{s}$ relaxation period and analyzed before and after sulfur bath therapy in comparison with normal healthy control. During the trial, the instrument was placed perpendicular to the anatomic area tested. A typical deformation curve is illustrated in (Figure 2). The following parameters were analyzed: immediate distension (Ue), delayed distension (Uv), final distension (Uf), immediate retraction (Ur), final retraction (Ua). $\mathrm{Ua} / \mathrm{Uf}$ represents the ratio of total retraction to total distension, called gross elasticity; $\mathrm{Ur} / \mathrm{Uf}$ represents the ratio of immediate retraction to total distension, called biological elasticity, while the ratio Uv/Ue represents the ratio of viscoelastic distension (5). The average values of two measurements were expressed in $\mathrm{mm}$ and used in subsequent calculations as previously reported by Dobrev in 2000 [13].

\subsection{Statistical Analysis}

Data were processed by Graph Pad Prism 5 statistical software. Two-way analysis of variance (ANOVA) followed by the post hoc Bonferroni's test were used for statistical analysis of data, which are presented as the mean \pm SEM. Values of $\mathrm{p}<0.05$ were considered significant.

\section{RESULTS}

\section{Evaluation of Skin Parameters}

In psoriatic plaques, we observed some skin deforma- tions suggested by changes of Skin elasticity and viscoelasticity (Figure 3).

In particular, we observed that the evaluation of biomechanical parameters is independent from the anatomical areas examined. The tested areas showed both a significative decrease of the elastic parameters (Uf, Ua, $\mathrm{Ur}, \mathrm{Ua} / \mathrm{Uf}, \mathrm{Ur} / \mathrm{Uf}$ ) and an high increase of the visoelastic ratio $(\mathrm{Uv} / \mathrm{Ue})$, in comparison with the healthy skin of control subjects. Sulfur bath therapy resulted in a significative improvement of the elastic and viscoelastic component with an increase in elasticity parameters and a decrease in viscoelasticity ratio (Figures 4 and 5).

\section{DISCUSSION}

Psoriasis is induced and maintained by multiple interactions between cells of the skin and immune system. It seems that the pathogenesis of psoriasis could involve a stage of cellular infiltration resulting in epidermal (keratinocyte) proliferation. The effects and efficacy of spa therapy in inflammatory skin diseases are only partly understood, and presumably incorporate chemical, thermal, mechanical and immunomodulatory effects $[19,20]$. Sulfur water can inhibit the production and/or release of cytokines, particularly IL-2 and interferon (IFN)- $\gamma$, from the Th1 lymphocytes subset which acts mainly on the $\mathrm{T}$ memory cells subset [21]. These latter lymphocytes are inhibited in their proliferation rate and in their ability to produce and release cytokines [22]. In addition the action of sulfur on the skin is thought to be due to its interaction with the cysteine and its catabolites contained in keratinocytes. The sulfur that penetrates the skin is oxidized and evokes various physiologic responses in the skin, such as vasodilation in the microcirculation and analgesic influence on the pain receptors [23]. Furthermore $\mathrm{HS}^{-}$

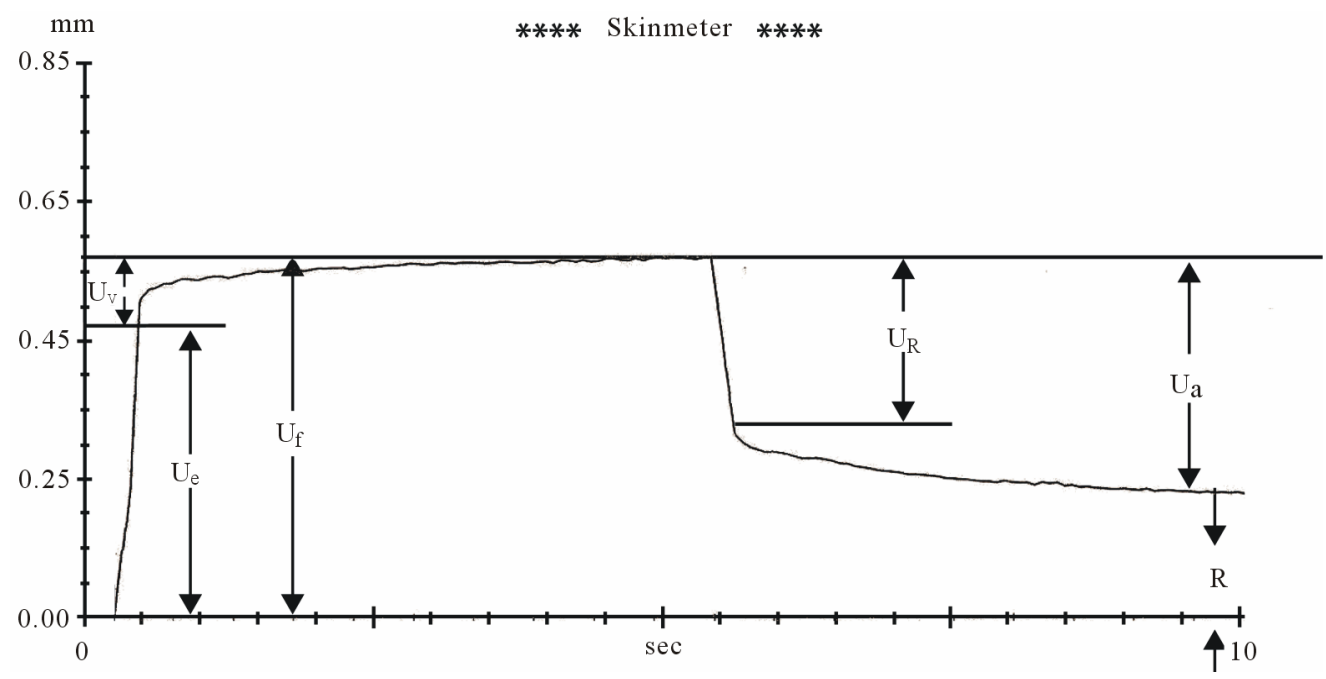

Figure 2. Tipical representation of skin deformation curve. Ue: immediate distension; Uv: delayed distension; Uf: final distension; Ur: immediate retraction; Ua: final retraction; R: residual deformation. 


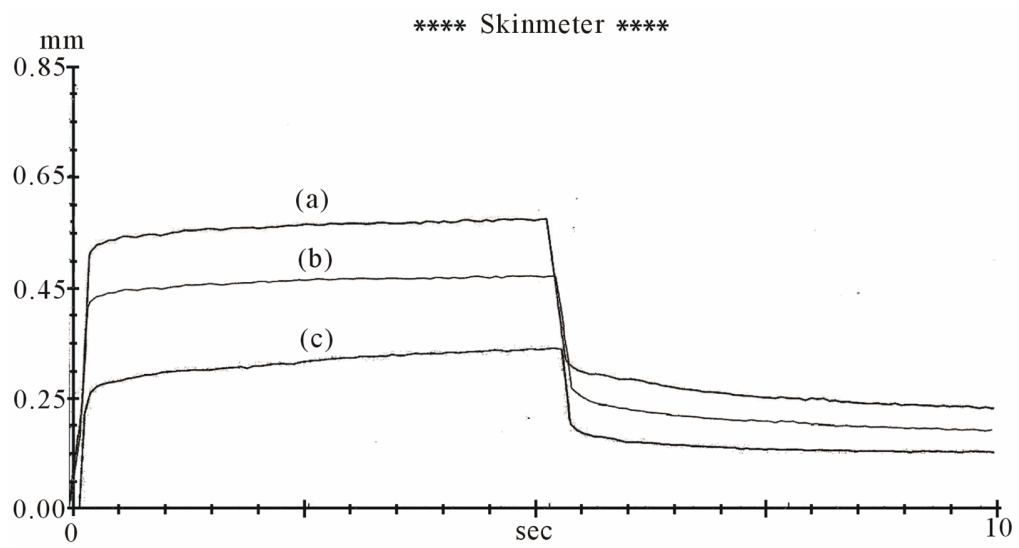

Figure 3. Representative skin deformation of psoriatic plaques: (a) Healthy skin (control); (b) Psoriatic plaques after sulfur bath therapy; (c) Psoriatic plaques before sulfur bath therapy.

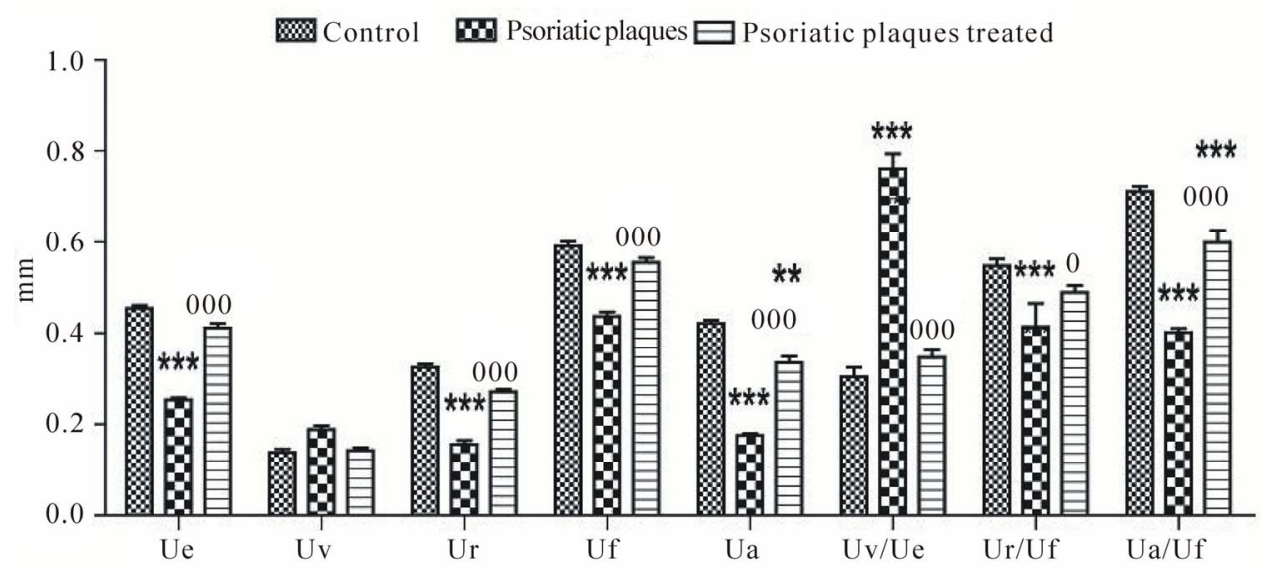

Figure 4. Biomechanical parameters (upper arm skin) of Control (healthy subjects), psoriatic plaques and psoriatic plaques treated with sulfur bath therapy. Two-way analysis of variance (ANOVA) followed by Bonferroni's test. ${ }^{* * *} \mathrm{p}<0.001$ significantly different from control; $\mathrm{p}<$ 0.05 and ${ }^{\circ 0} \mathrm{p}<0.001$ significantly different from psoriatic plaques $(\mathrm{n}=10)$.

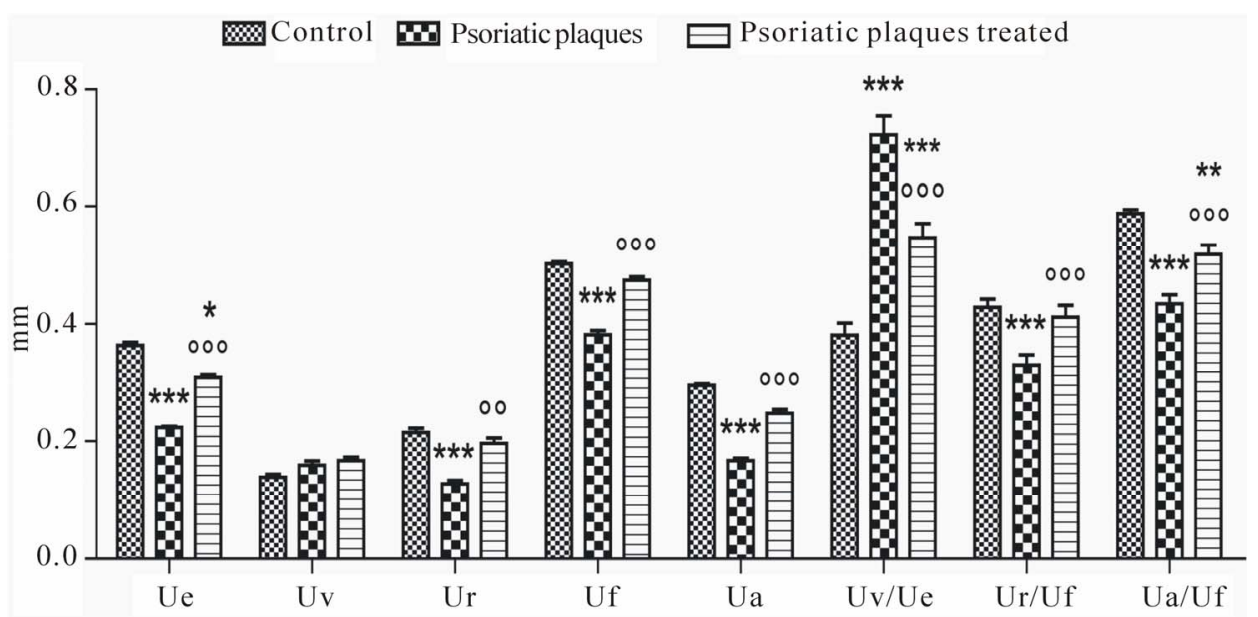

Figure 5. Biomechanical parameters (upper back skin) of Control (health subjects), of psoriatic plaques and psoriatic plaques treated with sulfur bath therapy. Two-way analysis of variance (ANOVA) followed by Bonferroni's test. ${ }^{*} \mathrm{p}<0.05$ and ${ }^{* * *} \mathrm{p}<0.001$ significantly different from control; ${ }^{\circ} \mathrm{p}<0.01$ and ${ }^{\circ 00} \mathrm{p}<0.001$ significantly different from psoriatic plaques $(\mathrm{n}=10)$. 
reduces the cellular cytotoxic response of peripheral blood lymphocytes as well as their production of IL-2, therefore de-activating the major players of local inflammatory response [24]. A high concentration of hydrogen sulfide combined with neutral $\mathrm{pH}$ (6.9 to 7.1) in the sulfur mineral water of Guardia Piemontese-Acquappesa, represent the optimal conditions for dermal absorption. In fact the rate of absorption of hydrogen sulfide is proportional to its concentrations; moreover, in neutral $\mathrm{pH}$ conditions hydrogen sulfide is present in its undissociated form $\left(\mathrm{H}_{2} \mathrm{~S}\right)$ that easily penetrates in the skin. The use of sulfur hypothermal water at $22^{\circ} \mathrm{C}$ for dilution of the sulfur hyper thermal water at $43^{\circ} \mathrm{C}$, dilutes the concentration of hydrogen sulfide about to $100 \mathrm{mg} / \mathrm{L}$ and this improves its bioavailability. In addition, the duration of the bath and its temperature influences the absorption of hydrogen sulfide and of other mineral substances, more than their concentration. Therefore, a temperature of about $34^{\circ} \mathrm{C}-35^{\circ} \mathrm{C}$ combined with a permanence time of $20 \mathrm{~min}$ in the tub are recommended to produce the best therapeutic results. This work demonstrates that the bath therapy with sulfur mineral water of Guardia Piemontese-Acquappesa (Italy), is able to restore the biomechanical properties of the skin. The analysis is founded on the measurements of the electromechanical transduction. A key finding of this work is the biological skin response of psoriasis patients by the Skin-meter. The increase of skin thickness as well as the degenerative changes in the fibrous network reported in psoriasis vulgaris cause a decrease in elasticity parameters $\mathrm{Ur}, \mathrm{Ua} / \mathrm{Uf}$ and $\mathrm{Ur} / \mathrm{Uf}$, with a decrease in the ability of the skin to return to its initial position after deformation [13]. Our results show that these values are significantly lower in psoriatic patients before sulfur bath therapy compared with normal healthy control. The parameters Uv, Ue are instead the portion of the viscoelasticity on the elastic part of the curve and are attributed to the displacement of the interstitial fluid, containing water and highly viscous glycosaminoglycans [25,26]. Particularly the Uv/Ue parameter was increased in psoriasis vulgaris, indicating the prevalence of the viscoelastic part over the elastic part of the deformation [13]. Sulfur bath therapy shows a reduction of the thinning psoriatic plaques. The main effects are showed by the following biomechanical parameters: 1) increase of the ratio $\mathrm{Ua} / \mathrm{Uf}$, that includes the viscous changes of the skin for both deformation and retraction, with the recovery of both elasticity of the skin's solid components (elastic fibers, etc.) and viscosity from the liquid content of the skin; 2) increase of the ratio $(\mathrm{Ur} / \mathrm{Uf})$, that includes measures of viscoelastic deformation and elastic retraction with the recovery the viscous and skin elasticity of psoriatic plaques; 3) decrease of the Uv/Ue that reflect a reduction of dermal oedema and the thinning of the psoriatic plaques. Also the changes in Uv/Ue can be used as indicators for the improvement of psoriasis [13]. This parameter is indicative of dermal water content [27], and reflects a restore of skin hydrating level, plasticity and electrical capacitance of the stratum corneum [28].

\section{CONCLUSION}

Sulfur bath therapy may have an important role in the therapeutic strategy of psoriasis, supporting and integrating the already consolidated pharmacological approaches. This study demonstrates the functionality of the Skin meter and confirms that measurements are not affected by the optical components of the circuitry. Therefore skin parameters measurements are not alterated by the presence of blood microcirculation in the subjects tested. Moreover, skin parameters are easily detected and analyzed and the data presented are in agreement with the results reported in previous studies [11-13,27,28]. We hypothesize that a practical implication is that this equipment shall be useful to get a direct monitoring of dermal skin conditions in relation to psoriasis skin disease.

\section{REFERENCES}

[1] Rashmi, R.K., Rao, S.J. and Basavaraj, K.H. (2009) A comprehensive review of biomarkers in psoriasis. Clinical and Experimental Dermatology, 34, 658-663. doi:10.1111/j.1365-2230.2009.03410.x

[2] Schon, M.P. and Boehncke, W.H. (2005) Medical progress: Psoriasis. New England Journal of Medicine, 352, 1899-1912.

http://www.nejm.org/doi/pdf/10.1056/NEJMra041320

[3] Mazzulla, S., Chimenti, R., Sesti, S., De Stefano, S., Morrone, M. and Martino, G. (2004) Effect of sulphurous Bioglea on psoriasis. Clinica Terapeutica, 155, 499-504.

[4] Mazzulla, S., Sesti, Martini, A., Nicoletta, V., S., De Stefano, S., Morrone, M. and Martino, G. (2011) Evaluation of natural reductants protecting red blood cell membranes against oxidative damage in psoriatic patients. Clinica Terapeutica, 162, 79-84. http://www.seuroma.it/clinica terapeutica/apps/autos.php ?id=882

[5] Yardley, H.J. and Summerly, R. (1981) Lipid composition and metabolism in normal and diseased epidermis. Pharmacology \& Therapeutics, 13, 357-383. doi:10.1016/0163-7258(81)90006-1

[6] Weger, W. (2010) Current status and new developments in the treatment of psoriasis and psoriatic arthritis with biological agents. British Journal of Pharmacology, 160, 810-820. doi:10.1111/j.1476-5381.2010.00702.x

[7] Pathirana, A., Ormerod, A.D., Saiag, P., Smith, C., Spuls, P.I., Nast, A. et al. (2009) European S3-guidelines on the systemic treatment of psoriasis vulgaris. Journal of the European Academy of Dermatology and Venereology, 23, S5-S70. doi:10.1111/j.1468-3083.2010.03671.x 
[8] Smith, C.H., Anstey A.V., Barker, J.N.W.N., Burden, A.D., Chalmers, R.J.G, Chandler, D., et al. (2009). British Association of Dermatologists guidelines for biologic interventions for psoriasis. British Journal of Dermatology, 161, 987-1019.

doi:10.1111/j.1365-2133.2009.09505.x

[9] Nagy Papp, K.A., Dekoven, J., Parsons, L., Pirzada, S., Robern, M., Robertson, L. and Tan, J.K. (2012) Biologic therapy in psoriasis: Perspectives on associated risks and patient management. Journal of Cutaneous Medicine and Surgery, 16, 153-168.

[10] Lukács, G.K., Sziray, A., Fazekas, K., Florián, A., Tamási, L. and Károlyi, Z. (2011). Adverse events during biological therapy-focusing on dermatological effects. Orvosi Hetilap, 152, 212-220.

[11] Ohshima, H., Kinoshita, S., Oyobikawa, M., Futagawa, M., Takiwaki, H., Ishiko, A. and Kanto, H. (2012) Use of Cutometer area parameters in evaluating age-related changes in the skin elasticity of the cheek. Skin Research and Technology, 19, 238-242.

doi:10.1111/j.1600-0846.2012.00634.x

[12] Tronnier, H., Wiebusch, M. and Heinrich, U. (2008) Change in skin physiological parameters in space-report on and results of the first study on man. Skin Pharmacology and Physiology, 21, 283-292. doi:10.1159/000148045.

[13] Dobrev, H. (2000) In vivo study of skin mechanical properties in psoriasis vulgaris. Acta Dermato-Venereologica, 80, 263-266. doi:10.1080/000155500750012135

[14] Ellis, C.N., Gorsulowsky, D.C., Hamilton, T.A., Billings, J.K., Brown, M.D., Headington, J.T., et al. (1986), Cyclosporine improves psoriasis in a double-blind study. JAMA, 256, 3110-3116. doi:10.1001/jama.1986.03380220076026

[15] Toichi, E., Torres, G., McCormick, T.S., Chang, T., Mascelli, M.A., Kauffmann C.L., et al. (2006). An antiIL-12p40 antibody down-regulates type 1 cytokines, chemokines, and IL-12/IL-23 in psoriasis. Journal of Immunology, 177, 4917-4926.

[16] Liu, Y., Krueger, J.G. and Bowcock, A.M. (2007) Psoriasis: Genetic associations and immune system changes. Genes \& Immunity, 8, 1-12. doi:10.1038/sj.gene.6364351

[17] Nickoloff, B.J., Qin, J.Z. and Nestle, F.O. (2007). Immunopathogenesis of psoriasis. Clinical Reviews in Allergy and Immunology, 33, 45-56.

\section{doi:10.1007/s12016-007-0039-2}

[18] Nestle, F.O., Kaplan, D.H. and Barker, J. (2009). Psoriasis. New England Journal of Medicine, 361, 496-509. doi:10.1056/NEJMra0804595

[19] Valitutti, S., Castellino, F. and Musiani, P. (1990) Effect of sulfurous (thermal) water on $\mathrm{T}$ lymphocyte proliferative response. Annals of Allergy, 65, 463-468.

[20] Celerier, P., Richard, A., Litoux, P. and Dreno, B. (1995) Modulatory effects of selenium and strontium salts on keratinocyte-derived inflammatory cytokines. Archives of Dermatological Research, 287, 680-682. doi:10.1007/BF00371742

[21] Magitz, H., Orion, E. and Wof, R. (2003) Balneotherapy in dermatology. Dermatologic Therapy, 16, 132-140. doi:10.1046/j.1529-8019.2003.01622.x

[22] Sainte-Laudy, J. (1987) Etude du pouvoir anti-degranulant de l'eau d'avene vis-à-vis de basophiles humains sensibilièe. International Journal of Immunotherapy, 4, 307-312.

[23] Nasermoaddeli, A. and Kagamimori, S. (2005), Balneotherapy in medicine: A rewiew. Environmental Health and Preventive Medicine, 10, 171-179. doi:10.1007/BF02897707

[24] Mirandola, P., Gobbi, G., Sponzilli, I. and Pambianco, M. (2007) Exogenous hydrogen sulfide induces functional inhibition and cell death of cytotoxic lymphocytes subsets. Journal of Cellular Physiology, 213, 826-833. doi:10.1002/jcp.21151

[25] Dobrev, H. (1999) In vivo study of skin mechanical properties in patients with systemic sclerosis. Journal of the American Academy of Dermatology, 40, 436-442. doi:10.1016/S0190-9622(99)70494-9

[26] Dobrev, H. (1998) In vivo study of skin mechanical properties in scleredema of Buschke. Acta Dermato-Venereologica, 78, 103-106. doi:10.1080/000155598433412

[27] Dobrev, H. (2000) Use of cutometer to assess epidermal hydration. Skin Research and Technology, 6, 239-244. doi:10.1034/j.1600-0846.2000.006004239.x

[28] Ryu, H.S., Joo, Y.H., Kim, S.O., Park, K.C. and Youn, S.W. (2008) Influence of age and regional differences on skin elasticity as measured by the Cutometer ${ }^{\circledR}$. Skin Research and Technology, 14, 354. doi:10.1111/j.1600-0846.2008.00302.x 